\title{
An inexpensive automated system for the measurement of rotational behavior in small animals
}

\author{
DAVID K. MCFARLANE, BUDA J. MARTONYI, and TERRY E. ROBINSON \\ University of Michigan, Ann Arbor, Michigan
}

\begin{abstract}
A reliable, low-cost automated system for the quantification of rotational behavior from up to 10 subjects simultaneously is described. The system resolves quarter and full turns to the left and right, and can relay data to another computer for statistical analysis. The system is easily constructed from simple and inexpensive components, including the computer, that total less than $\$ 1,000$ and can be used with a variety of different test chambers.
\end{abstract}

Automated systems for measuring animal rotations, often called rotometers, have been in use since 1970 (Ungerstedt \& Arbuthnott, 1970), and a number of commercial and lab-built systems have been described (Barber, Blackburn, \& Greenwood, 1973; Bonatz, Steiner, \& Huston, 1987; Greenstein \& Glick, 1975; Jerussi, 1982; Schmidt \& Dubach, 1988; Schwarz, Stein, \& Bernard, 1978; Yehuda \& Wurtman, 1975; Walsh \& Silbergeld, 1979). However, commercial systems are very expensive and some lab-built systems require relatively expensive acquisition hardware and dedicated PCs. We developed an automated data collection system based on the inexpensive Commodore 64 , which comes with an array of acquisition hardware built-in, and its capability was easily extended by the use of an inexpensive commercial $1 / 0$ board. Sensor units were also developed, and these resolve every $90^{\circ}$ movement and fit over a variety of test chambers. The flexible software allows the acquisition of data simultaneously from up to 10 different rotometers, and then data can be transmitted to a more sophisticated computer for statistical analysis. The cost of the hardware for the entire 10-unit system, including the Commodore 64 , is less than $\$ 1,000$, and it is easily constructed from readily available components. Alternatively, the sensor units could be added to a system built by using any PC and appropriate $\mathrm{I} / \mathrm{O}$ hardware, and the software could be adapted from the following description.

We are indebted to Chris Leiby for his earlier rotometer software on the Rockwell AIM 65. This work was supported by Grant DA04294 to T.E.R. and USPHS NS 22157 to Jill B. Becker, and portions of it were presented at the Annual Meeting of the Society for Neuroscience (McFarlane, Becker, Martonyi, \& Robinson, 1990). Requests for reprints should be addressed to T. Robinson, University of Michigan, 3064 Neuroscience Bldg., 1103 E. Huron St., Ann Arbor, MI 48109-1687. Inquiries regarding the system should be sent to D. McFarlane, SmartTech Systems Engineering, 1293 Orlando Dr., Haslett, MI 48840 (e-mail:dkmlcc@lcc.edu.).

\section{HARDWARE}

Figure 1 shows a block diagram of the hardware for a 10-unit automated rotometer system. Briefly, the system consists of a Commodore 64 computer and peripherals (video monitor, disk drive, printer, and RS-232 adapter), an SS100 Plus Simplified Digital I/O Board, sensor units, a routing and distribution box (not shown), external 5-VDC power supply, and connecting cables. The Commodore 64 computer provides a slightly modified 6502 processor running at $1 \mathrm{MHz}, 64 \mathrm{~K}$ of $\mathrm{RAM}, \mathrm{I} / \mathrm{O}$ ports and timers on two 6526 complex interface adapters (CIA), and a 6581 sound interface device (SID). The automated rotometer software uses the time-of-day (TOD) timer on CIA 2 to time acquisition epochs and uses the SID to supply white noise through an audio loudspeaker (usually part of the monitor).

Rotation of the subject is detected and encoded by a sensor unit suspended over the testing chamber. The sensor unit consists of a custom-built 2-bit quadrature, 4-position optical encoder powered by an external 5-VDC power supply: A 3-cm long axle and bushing derived from common model aircraft supplies is mounted partially inside a simple plastic enclosure so that the axle extends outside; the subject can be attached to this end by means of a flexible wire cable (model aircraft cable) with claw clips on each end that fasten either to the subject's harness or to a clasp soldered onto the end of the axle. The internal end of the axle is soldered to a 2-cm disk that is half black and half white. A circuit board directly above the disk holds two reflective-object detectors (ROD) separated by $90^{\circ}$. Each ROD contains its own LED light source and photodetector, which allows it to detect whether it is above a light or a dark sector of the disk, and together the pair provides an encoded signal to the computer indicating the direction the subject faces as it turns, as is illustrated in Figure 2. Higher rotational resolution could be achieved with the same hardware simply by dividing the disk into more sectors and properly placing the two photocells, al- 


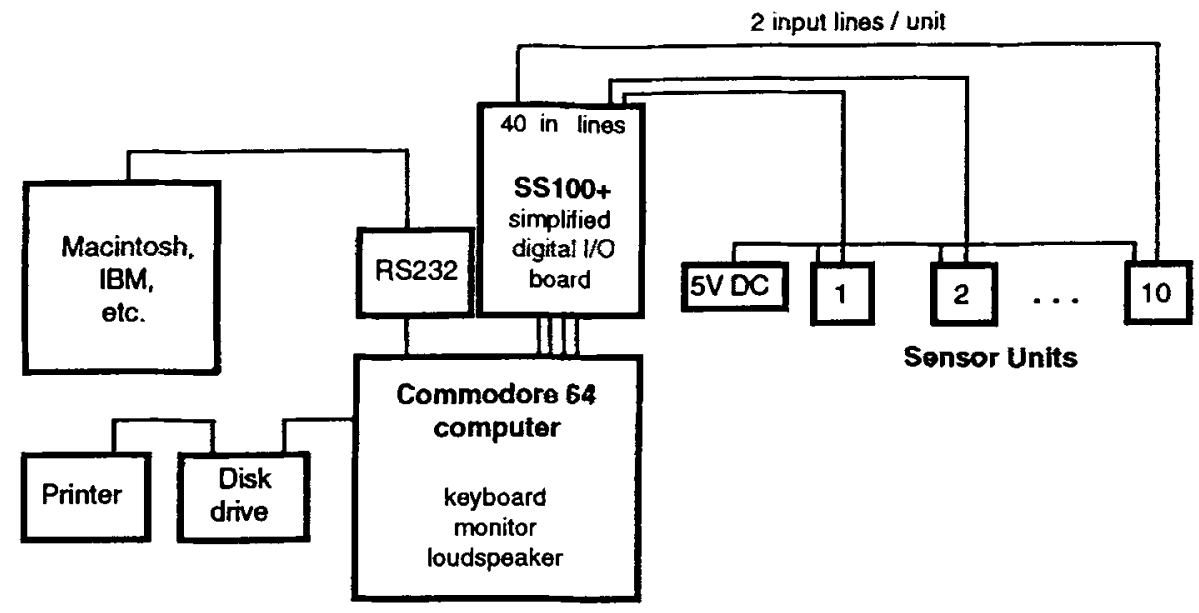

Figure 1. Block diagram of rotometer system hardware.

though this would reduce the maximum rotational rates and total number of epochs acquired, and the software must be changed to process the desired resolution. Two trim-pots are used to adjust the sensitivity of the photodetectors to produce a solid transition from logic "high" to "low" when the disk under them turns from black to white. This circuit is connected to the computer over cables up to $20 \mathrm{ft}(7 \mathrm{~m})$ in length, with no signal conditioning other than that provided by the I/O board. All input is memory-mapped to 8-bit ports, and groups of four or fewer sensor units are connected to one port so that the D1 signal of each unit connects to the first available evennumbered bit of the port and its D2 signal connects to the next bit (Figures 2 and 3 ).

The single user port of the C- 64 could serve four such sensor units (provided the software input address is changed to the user port), but to extend this the SS100 Plus Simplified Digital I/O Board was used. (The board

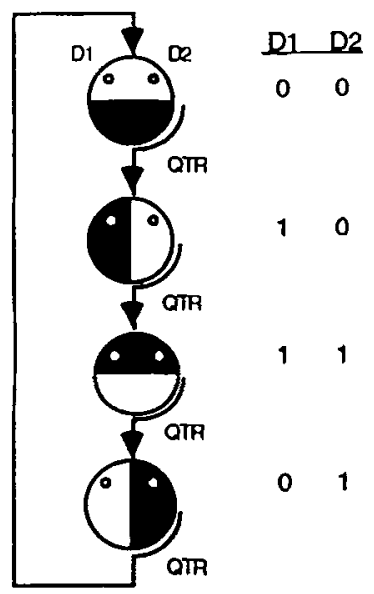

Figure 2. Principle of sensor unit encoding. D1 and D2 are the two photodetectors placed above the light/dark disk in the sensor unit. The columns on the right show the encoded signal from the detector pair as the disk rotates through the four quarter turns in the illustration on the left. is available from Schnedler Systems, 25 Eastwood Rd., P.O. Box 5964, Asheville, NC 28813, telephone 704-2744646.) This board provides 40 lines of digital input and 40 lines of digitally switched output, each arranged as five 8-bit ports; the rotometer software uses 20 of the input lines ( 2.5 ports) to support 10 sensor units. More units could be supported by this board, but this limit was chosen both in consideration of operator interventions that occur between acquisition periods and to allow 100 epochs of data to fit within a secure area of RAM (discussed below).

\section{SOFTWARE}

The software consists of an acquisition routine and a user-interface "shell." The acquisition routine is written in machine language, but the rest of the program is written in Commodore BASIC 2.0. In the interest of brevity, and because others may wish to institute their own user-interface shell around the acquisition routine, the BASIC shell program is only briefly described here. Complete source code is available from the authors on request.

\section{Acquisition Routine}

The acquisition routine accumulates counts of quarter and full turns in each direction (one full turn is four consecutive quarter turns in the same direction) into 8-bit locations in a fixed area of RAM during one acquisition epoch or "interval" that may last from $1 \mathrm{sec}$ to $13 \mathrm{~h}$. It is the job of the calling program to move the data to some other safe location between epochs and to call the acquisition routine again until all epochs are completed for the test period. The TOD alarm and the number of rotometers (n.rot) must also be set by the calling program before calling the acquisition routine.

Each rotometer uses only two bits, and, therefore, when the acquisition routine reads one byte of input, it gets data from four rotometers at once. In brief, the routine begins by starting up the TOD clock, reading in a set of four 


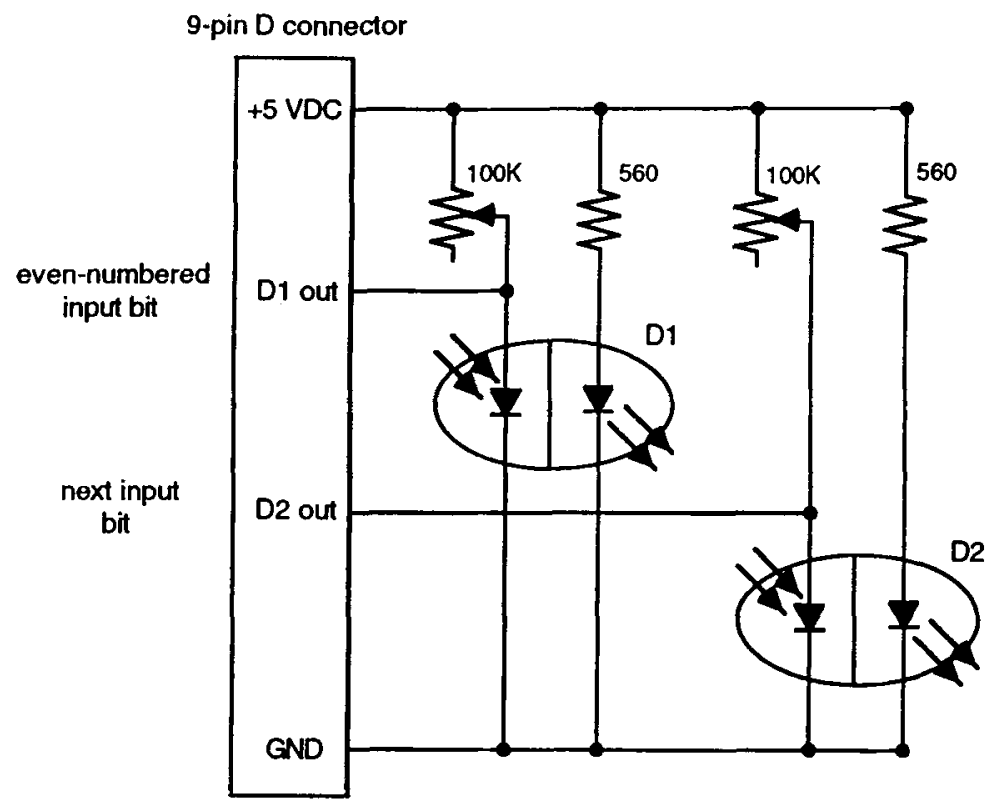

Figure 3. Sensor unit schematic. D1 and D2 are Optek OPB730, placed $90^{\circ}$ apart within the radius of the rotating disk. $100 \mathrm{~K}$ pots are on-board trim-pots.

rotometers from the $\mathrm{I} / \mathrm{O}$ board, processing the input from this set, and then going on to the next set of four until it has scanned all active rotometers, after which it begins with the first set again. It continues doing this until the TOD alarm goes off-this is checked after each pass through the set of active rotometers.

This routine achieves a sampling rate of $833 \mathrm{~Hz}$ in benchmark tests in which the routine is modified to acquire data from 10 rotometers simulated in RAM. This rate will keep up with rotational rates up to 208 rotations/ $\mathrm{sec}$, as long as the counters do not overflow. Also, suspending system interrupts may aid in the smooth operation of this routine while data are acquired.

\section{Shell}

The shell program completes the software by getting experimental parameters for the session from the user, properly calling the acquisition routine to complete the test session, producing a report of the results of the session, and possibly storing the data to disk at the end of a session for later reference and analysis. The shell can transmit the completed data to another computer for more extensive analysis, with the rotometer computer acting as a low-cost but able "slave" producing results for an advanced "master." When transmitting data, tabulated numbers are separated with $\langle$ tab $\rangle$ characters to aid entry to most spreadsheet programs. This shell is written completely in BASIC and provides a menu-driven functionkey interface for performing these functions. Figure 4 shows a block diagram of the subroutine modules provided by the shell.

There is no pause between epochs, and the acquired data are stored in a secure area of RAM (\$C000-\$CFFF) that is undisturbed by all normal operations of the operating system, including a warm reboot and the BASIC "run" command (Commodore Business Machines, 1982). Thus, the data are impervious to crashes and hang-ups. This makes the system robust in its ability to recover data in the rare event of a system crash or malfunction. At 4 bytes/subject epoch, 100 epochs of 10 subjects fit snugly into this space, and these epochs can be divided among several experimental treatment periods. Greater data security could be achieved by modifications to save data to disk at the end of each epoch. This would produce a pause of 1-8 sec between each epoch.

Since the acquisition routine counts only 255 (\$FF) turns into the accumulators during any one epoch before restarting at 0 , the quarter-turn count is used as only an indicator of extra quarter turns, and the true quarter turns are calculated using the formula

true quarter turns $=q t+h-256 \cdot\{q t<[(4 \cdot f t)-h]\}$, where $q t=$ quarter turns counted, $f t=$ full turns counted, and $h=256 \cdot \operatorname{INT}(f t / 64)$. If larger counts are needed, the acquisition code could be modified easily to use twobyte accumulators. This would halve the allowable number of total epochs in the allotted RAM (or double the memory requirement for the same number of epochs) but would allow a maximum of $64 \mathrm{~K}$ turns to be counted during each epoch.

\section{AVAILABILITY}

A binary file containing the source code may be obtained from the author by e-mail (gb92@um.cc.umich.edu), or a Commodore-64-compatible disk containing the software described may be obtained by sending a 5.25-in. DSDD disk with postage-paid return mailer to the authors. 


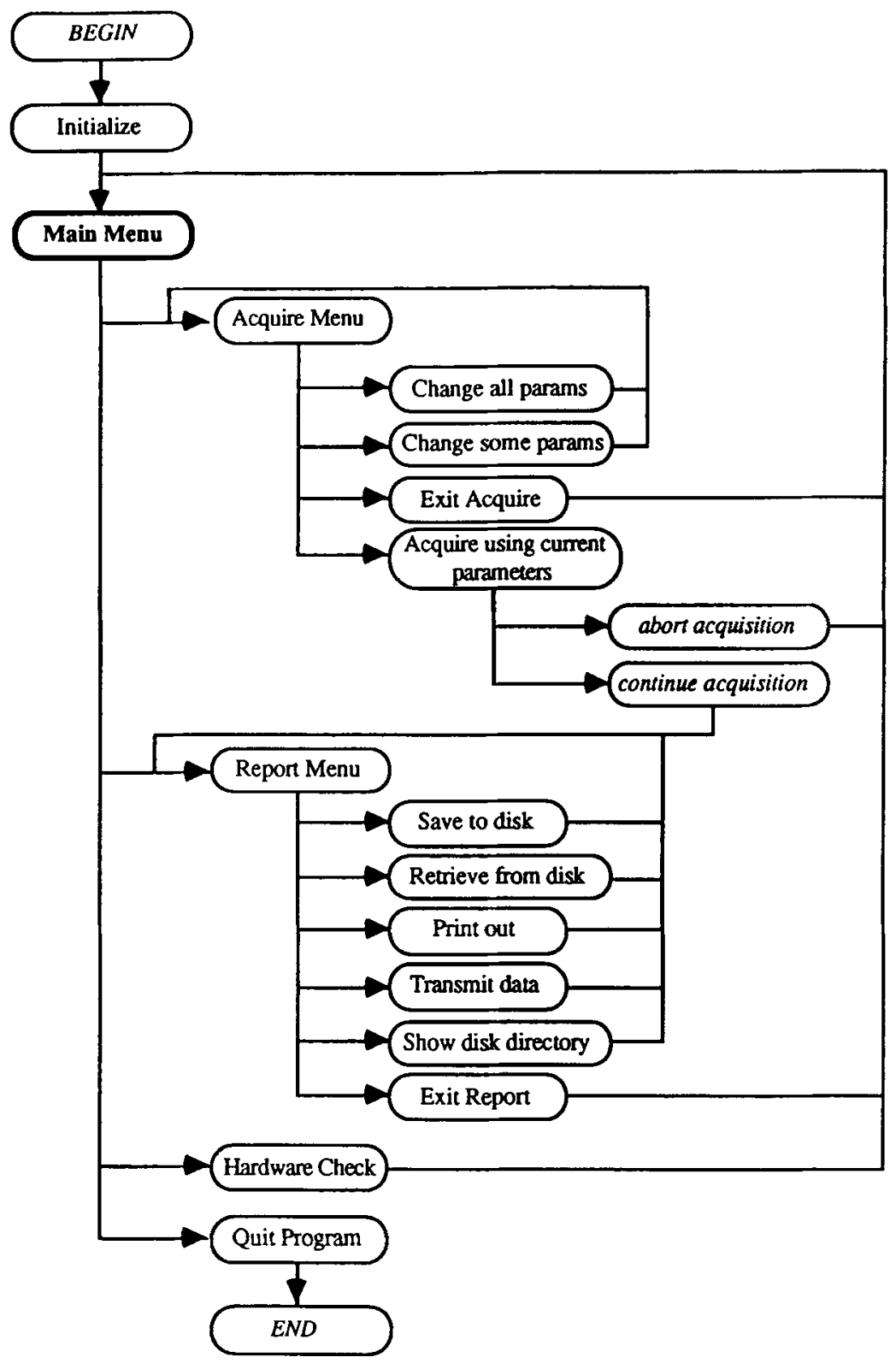

Figure 4. Block diagram of subroutine modules in the rotometer software shell.

\section{REFERENCES}

Barber, D. L., Blackburn, T. P., \& Greenwood, D. T. (1973). An automated apparatus for recording rotational behavior in rats with brain lesions. Physiology \& Behavior, 11, 117-120.

Bonatz, A. E., Steiner, H., \& Huston, J. P. (1987). Video image analysis of behavior by microcomputer: Categorization of turning and locomotion after 6-OHDA injection into the substantia nigra. Journal of Neuroscience Methods, 22, 13-26.

COMMODORE BUSINESS MACHINES (1982). Commodore 64 programmer's reference guide. Indianapolis: Howard W. Sams.

Greenstein, S., \& GLick, S. D. (1975). Improved automated appara- tus for recording rotation (circling behavior) in rats or mice. Pharmacology, Biochemistry \& Behavior, 3, 507-510.

Jerussi, T. P. (1982). A simple, inexpensive rotometer for automatically recording the dynamics of circling behavior. Pharmacology, Biochemistry \& Behavior, 16, 353-357.

McFarlane, D. K., Martonyi, B. J., Becker, J. B., \& Robinson, T. E. (1990). An inexpensive automated system for the quantification of rotational behavior in small animals. Society for Neuroscience Abstracts, 16, 754.

Schmidt, R. H., \& Dubach, M. D. (1988). A computer-based rotation and activity monitor for non-human primates and other animals. Journal of Neuroscience Methods, 24, 243-251.

SchWarz, R. D., SteIn, J. W., \& Bernard, P. (1978). Rotometer for 
recording rotation in chemically or electrically stimulated rats. Physiology \& Behavior, 20, 351-354.

UNGERSTEDT, U., \& ARBUTHNOTT, G. W. (1970). Quantitative recording of rotational behavior in rats after 6-hydroxy-dopamine lesions of the nigrostriatal dopamine system. Brain Research, 24, 485-493.

Walsh, M. J. \& Silbergeld, E. K. (1979). Rat rotation monitoring for pharmacology research. Pharmacology, Biochemistry \& Behavior, $10,433-436$.

YehUda, S., \& WuRTMan, R. J. (1975). Dopaminergic neurons in the nigro-striatal and mesolimbic pathways: Modulation of specific effects of d-amphetamine. European Journal of Pharmacology, 30, 154-158.

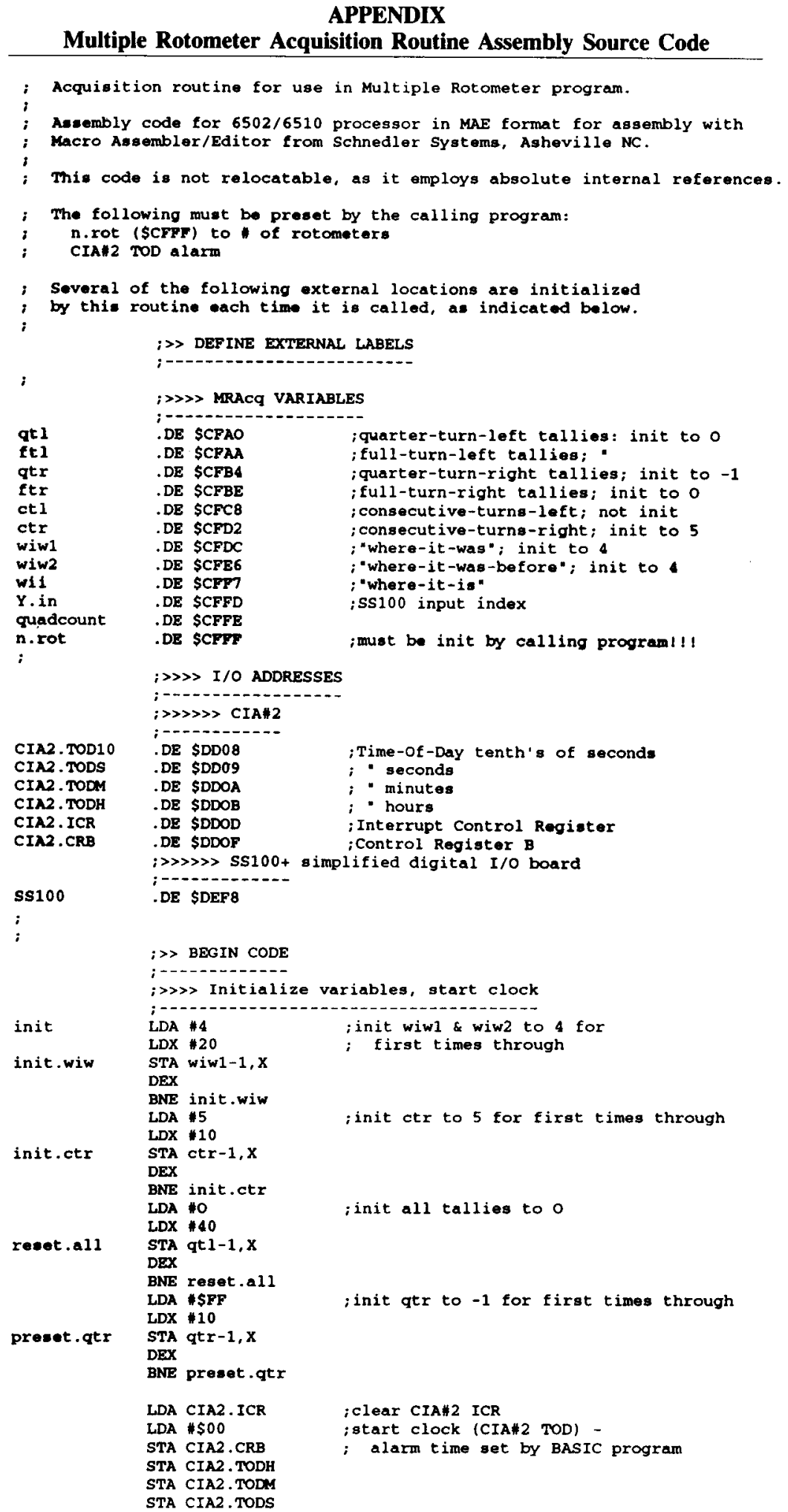

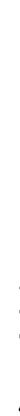

preset.qtx 


\section{APPENDIX (Continued)}

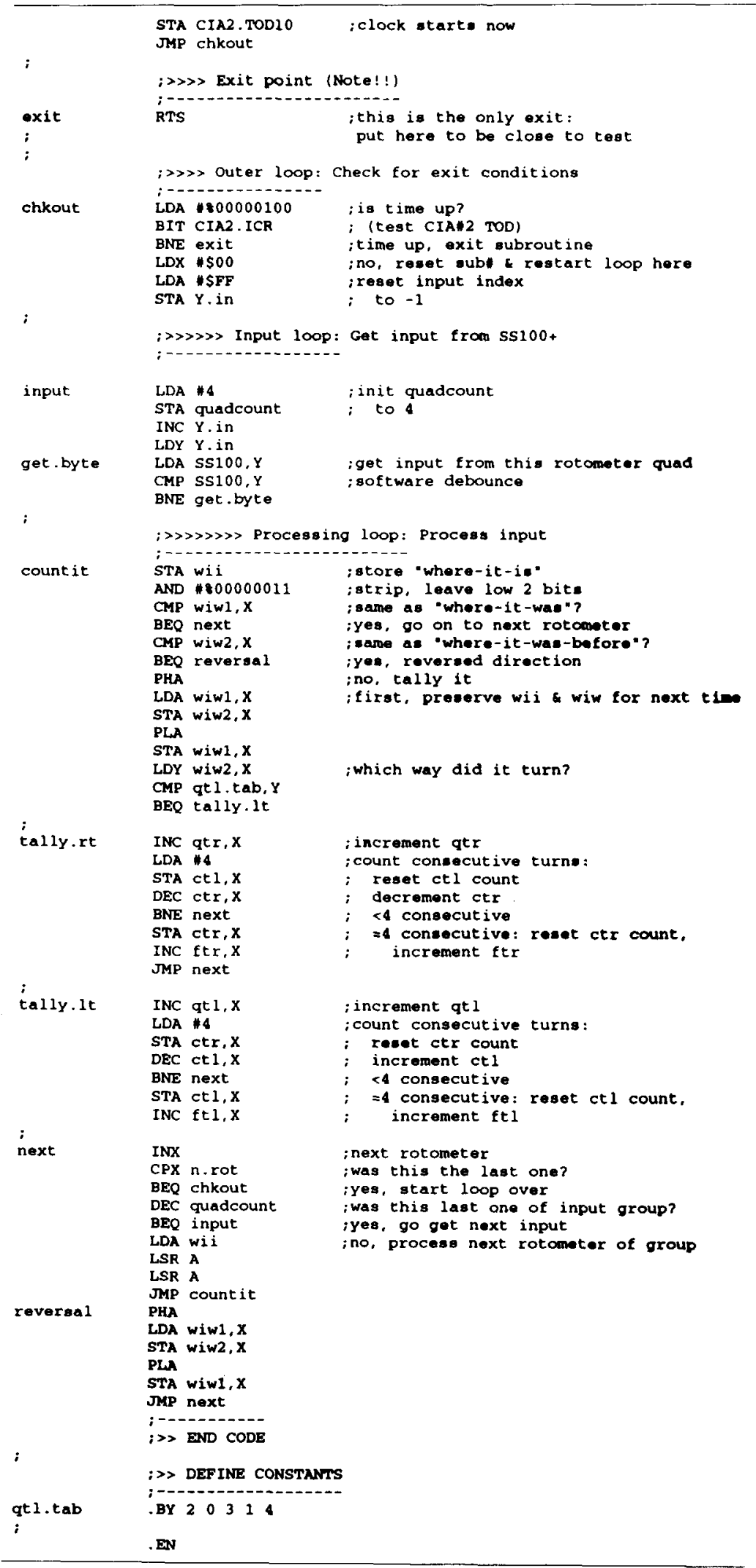

(Manuscript received October 22, 1991;

revision accepted for publication March 24, 1992.) 